\title{
Tecnologias educacionais aplicadas à saúde sexual e reprodutiva dos adolescentes de escolas públicas
}

\author{
Educational technologies applied to sexual and reproductive health of adolescents from \\ public schools
}

Tecnologías educativas aplicadas a la salud sexual y reproductiva de los adolescentes en las escuelas públicas

Sibele Naiara Ferreira Germano ${ }^{1 *}$, Alex Araújo Rodrigues ${ }^{1}$, Flávia Brito Bessa1, Paulo Henrique Lira Matos $^{1}$, Silvane e Silva Evangelista ${ }^{1}$.

\section{RESUMO}

Objetivo: Identificar na literatura as tecnologias educacionais aplicadas nas escolas públicas para a saúde sexual e reprodutiva dos adolescentes. Métodos: Trata-se de uma Revisão Integrativa de Literatura, com buscas em março a abril de 2020, em quatro bases de dados: Literatura Latino-Americana e do Caribe em Ciências da Saúde, Medical Literature Analysis and Retrieval System Online, Índice Bibliográfico Espanhol em Ciências da Saúde e Banco de Dados em Enfermagem. Resultados: Identificou-se 753.567 artigos acessados nas bases de dados mencionando um dos descritores: Adolescente, Saúde sexual e reprodutiva, Tecnologia educational, Serviços de saúde escolar. Após o cruzamento destes descritores identificou-se 147 artigos que passaram por uma triagem aplicando-se os critérios de exclusão, através da releitura dos títulos e resumos selecionados, excluíram-se 135 artigos, resultando 12 artigos que atenderam os critérios de inclusão da pesquisa e responderam à questão norteadora, estes foram lidos na integra fazendo parte da amostragem final da pesquisa. Considerações finais: Os estudos demonstram uma variada quantidade de tecnologias educacionais para os adolescentes sobre saúde sexual e reprodutiva a serem utilizadas nas escolas públicas, e enfatizam o espaço escolar como uma potencialidade para se trabalhar essas tecnologias educacionais.

Palavras-chave: Adolescente, Saúde sexual e reprodutiva, Tecnologia educacional, Serviços de saúde escolar.

\section{ABSTRACT}

Objective: Identify in the literature the educational technologies applied in public schools for the sexual and reproductive health of adolescents. Methods: This is an Integrative Literature Review, with searches from March to April 2020, in four databases: Latin American and Caribbean Literature in Health Sciences, Medical Literature Analysis and Retrieval System Online, Spanish Bibliographic Index in Health Sciences and Nursing Database. Results: 753.567 articles were identified in the databases, mentioning one of the descriptors: Adolescent, Sexual and reproductive health, Educational technology, School health services. After crossing these descriptors, 147 articles were identified that were screened using the exclusion criteria, through the rereading of the selected titles and abstracts, 135 articles were excluded, resulting in 12 articles that met the inclusion criteria of the research and answered the guiding question, these were read in full as part of the final survey sample. Final considerations: The studies demonstrate a varied amount of educational technologies for adolescents on sexual and reproductive health to be used in public schools and emphasize the school space as a potential to work with these educational technologies.

Keywords: Adolescent, Sexual and reproductive health, Educational technology, School health services.

${ }^{1}$ Escola de Saúde Pública (ESAP) / Secretaria Municipal de Saúde de Manaus (SEMSA). Universidade do Estadual do Amazonas (UEA). Curso de especialização em Saúde Pública com Ênfase em Estratégia Saúde da Família. Manaus - AM *E-mail: sibelenaiaraferreiragermano@gmail.com 


\section{RESUMEN}

Objetivo: Identificar en la literatura las tecnologías educativas aplicadas en las escuelas públicas para la salud sexual y reproductiva de los adolescentes. Métodos: Esta es una revisión de literatura integradora, con búsquedas de marzo a abril de 2020, en cuatro bases de datos: literatura latinoamericana y caribeña en ciencias de la salud, análisis de literatura médica y sistema de recuperación en línea, índice bibliográfico en español en Ciencias de la Salud y Base de Datos de Enfermería. Resultados: Se identificaron 753,567 artículos en las bases de datos, mencionando uno de los descriptores: adolescente, salud sexual y reproductiva, tecnología educativa, servicios de salud escolar. Después de cruzar estos descriptores, se identificaron 147 artículos que se examinaron utilizando los criterios de exclusión, a través de la relectura de los títulos y resúmenes seleccionados, se excluyeron 135 artículos, lo que resultó en 12 artículos que cumplieron con los criterios de inclusión de la investigación y respondió la pregunta guía, estos se leyeron en su totalidad como parte de la muestra final de la encuesta. Consideraciones finales: Los estudios demuestran una cantidad variada de tecnologías educativas para adolescentes sobre salud sexual y reproductiva que se utilizarán en las escuelas públicas, y enfatizan el espacio escolar como un potencial para trabajar con estas tecnologías educativas.

Palabras clave: Adolescente, Salud sexual y reproductiva, Tecnología educativa, Servicios de salud escolar.

\section{INTRODUÇÃO}

As Tecnologias Educacionais (TEs) no âmbito da saúde são estratégias inovadoras que surgiram com a globalização para uma educação mais dinâmica e ativa, fortalecendo a parceria escola e serviços de saúde, com os adolescentes, família e comunidade. No entanto, um desafio emergente é a necessidade de ampliar o acesso destas TE com informações sobre saúde sexual e reprodutiva para todos os adolescentes de escolas públicas, que apresentam maiores vulnerabilidades devido o contexto socioeconômico e cultural em que vivem (SILVA DML, et al., 2017).

A adolescência é definida pela Organização Mundial da Saúde (OMS) como o período entre a infância e a idade adulta, tendo como parâmetro entre os 10 aos 19 anos de idade, o Ministério da Saúde (MS) considera esses mesmos período, porém o Estatuto da Criança e do Adolescente (ECA) diverge ao adotar como critério o período de 12 a 19 anos de idade (GONÇALVES LFF, et al., 2016).

Nesse período é preciso que estes indivíduos sejam inseridos em um ambiente de reflexões e discussões sobre as questões inerentes a sua sexualidade e reprodução. (FIEDLER MW, et al., 2015). Situações como a gravidez na adolescência, sexualidade, Infecções Sexualmente Transmissíveis (ISTs) são muito frequentes e podem ser trabalhados a partir do uso das TE em saúde, uma vez que estes instrumentos tecnológicos fazem parte do cotidiano destes adolescentes (SILVA DML, et al., 2017).

O ambiente escolar é considerado o espaço ideal para a utilização dessas tecnologias como estratégias para a promoção da saúde e prevenção das doenças na adolescência, dada a sua capacidade de abrangência sociocultural, uma vez que atitudes e valores são formados nesse ambiente, onde as atividades educativas em saúde devem estar associadas a uma educação construtiva, ativa e dialógica que promova o acolhimento e empoderamento dos adolescentes para o autocuidado (SFAIR SC, et al., 2015).

Tendo em vista a vulnerabilidade da adolescência, devido os aspectos emocionais e socioculturais, que caracterizam este período como necessário a orientação e atenção especial, destaca-se a importância da construção de estratégias entre a equipe de saúde dos serviços de saúde e os professores das escolas para a promoção da saúde, prevenção de doenças e acompanhamento saudável das modificações que ocorrem neste período (BRASIL, 2018).

Diante do exposto, surgiu a seguinte questão de pesquisa "Quais as evidências disponíveis na literatura, acerca das tecnologias educacionais sobre saúde sexual e reprodutiva dos adolescentes nas escolas públicas vinculadas aos serviços de saúde, nos últimos 5 anos (2014 a 2019)?”. Assim o objetivo desta pesquisa foi identificar na literatura as tecnologias educacionais aplicadas nas escolas públicas para a saúde sexual e reprodutiva dos adolescentes. 


\section{MÉTODOS}

Trata-se de uma Revisão Integrativa de Literatura (RIL), a questão de pesquisa passou pela a estratégia PICO, um acrônimo no idioma inglês que significa " $\mathrm{P}$ - Population (população), I - Intervention (intervenção), C - Comparison (comparação) e O - Outcome (resultados)" (DONATO H e DONATO M, 2019). Utilizando esta estratégia conferiu-se P - aos adolescentes de escolas públicas, I - aos Serviços de saúde na escola, C aos adolescentes de escola pública com acesso as tecnologias educacionais, $R$ a saúde sexual e reprodutiva dos adolescentes, que resultou na seguinte questão norteadora: Quais as evidências disponíveis na literatura, acerca das tecnologias educacionais sobre saúde sexual e reprodutiva dos adolescentes nas escolas públicas vinculadas aos serviços de saúde, nos últimos 5 anos (2014 a 2019)?

A busca ocorreu em março a abril de 2020 , em quatro bases de dados para a seleção das publicações, a saber: LILACS (Literatura Latino-Americana e do Caribe em Ciências da Saúde), MEDLINE (National Library of Medicina), IBECS (Índice Bibliográfico Espanhol em Ciências da Saúde), e BDENF (Banco de Dados em Enfermagem). Os descritores utilizados foram: Adolescente (teenager), Saúde sexual e reprodutiva (Sexual and reproductive health), Tecnologia educacional (educational technology), Serviços de saúde escolar (school health services), com o operador booleano AND (Tabela 1).

Tabela 1 - Cruzamento dos descritores de acordo com as bases de dados de março a abril de 2020. Manaus, AM, Brasil. $(n=12)$.

\begin{tabular}{|c|c|c|c|c|c|c|c|c|c|c|}
\hline \multirow[t]{2}{*}{$\begin{array}{l}\text { Base de } \\
\text { dados e } \\
\text { estratégias }\end{array}$} & \multirow[t]{2}{*}{$\begin{array}{l}\text { Cruzamento dos } \\
\text { descritores }\end{array}$} & \multirow[t]{2}{*}{$\begin{array}{l}\text { Resultado } \\
\text { da Busca }\end{array}$} & \multirow{2}{*}{$\begin{array}{c}\text { Pré-seleção } \\
\text { com } \\
\text { critérios da } \\
1 \text { exclusão } \\
\text { Ú5a }\end{array}$} & \multicolumn{6}{|c|}{$\begin{array}{l}\text { Artigos após leitura de } \\
\text { título e resumos com } \\
\text { outros critérios de exclusão }\end{array}$} & \multirow[t]{2}{*}{$\begin{array}{l}\text { Artigos } \\
\text { Inclusos }\end{array}$} \\
\hline & & & & NA & NI & NP & NT & NQ & ER & \\
\hline \multicolumn{11}{|l|}{ LILACS } \\
\hline E1 & $\begin{array}{c}\text { "D1"AND“D2" } \\
\text { AND“D3"AND“D4" }\end{array}$ & 0 & 0 & 0 & 0 & 0 & 0 & 0 & 0 & 0 \\
\hline E2 & "D1"AND“D2" & 96 & 33 & 5 & 0 & 1 & 7 & 10 & 3 & 7 \\
\hline E3 & “D2"AND“D3" & 5 & 4 & 1 & 0 & 0 & 1 & 1 & 0 & 0 \\
\hline $\mathrm{E} 4$ & “3” AND “4 & 1 & 1 & 1 & 0 & 0 & 0 & 0 & 0 & 0 \\
\hline E5 & “D1"AND“D2"AND“D3” & 0 & 0 & 0 & 0 & 0 & 0 & 0 & 0 & 0 \\
\hline \multicolumn{11}{|l|}{ MEDLINE } \\
\hline $\mathrm{E} 1$ & $\begin{array}{c}\text { "D1"AND“D2" } \\
\text { AND“D3"AND“D4" }\end{array}$ & 0 & 0 & 0 & 0 & 0 & 0 & 0 & 0 & 0 \\
\hline E2 & "D1"AND“D2" & 0 & 0 & 0 & 0 & 0 & 0 & 0 & 0 & 0 \\
\hline E3 & “D2"AND“D3" & 0 & 0 & 0 & 0 & 0 & 0 & 0 & 0 & 0 \\
\hline $\mathrm{E} 4$ & “D3”AND“D4” & 5 & 0 & 0 & 0 & 0 & 0 & 0 & 0 & 0 \\
\hline E5 & “D1"AND“D2"AND“D3" & 0 & 0 & 0 & 0 & 0 & 0 & 0 & 0 & 0 \\
\hline \multicolumn{11}{|l|}{ IBECS } \\
\hline $\mathrm{E} 1$ & $\begin{array}{c}\text { "D1"AND“D2" } \\
\text { AND“D3"AND“D4" }\end{array}$ & 0 & 0 & 0 & 0 & 0 & 0 & 0 & 0 & 0 \\
\hline E2 & "D1"AND“D2" & 5 & 5 & 0 & 0 & 0 & 1 & 4 & 0 & 0 \\
\hline E3 & “D2”AND“D3" & 0 & 0 & 0 & 0 & 0 & 0 & 0 & 0 & 0 \\
\hline $\mathrm{E} 4$ & "3" AND "4" & 3 & 3 & 0 & 0 & 0 & 2 & 0 & 0 & 1 \\
\hline E5 & “D1"AND“D2”AND“D3” & 0 & 0 & 0 & 0 & 0 & 0 & 0 & 0 & 0 \\
\hline \multicolumn{11}{|l|}{ BDENF } \\
\hline $\mathrm{E} 1$ & $\begin{array}{c}\text { "D1"AND“D2" } \\
\text { AND“D3"AND“D4" }\end{array}$ & 0 & 0 & 0 & 0 & 0 & 0 & 0 & 0 & 0 \\
\hline E2 & "D1"AND“D2" & 27 & 11 & 0 & 0 & 1 & 4 & 1 & 2 & 3 \\
\hline E3 & "D2"AND“D3" & 1 & 1 & 0 & 0 & 0 & 0 & 0 & 1 & 0 \\
\hline E4 & “D3”AND“D4” & 3 & 3 & 0 & 0 & 0 & 2 & 0 & 1 & 0 \\
\hline E5 & “D1"AND“D2"AND“D3" & 1 & 1 & 0 & 0 & 0 & 0 & 0 & 0 & 1 \\
\hline
\end{tabular}

Legenda: E - (Estratégia); "D1" - (Descritor "Adolescente"); "D2" - (Descritor "Saúde Sexual e Reprodutiva"); "D3" - (Descritor "Tecnologia Educacional"); "D4" - (Descritor "Serviços de Saúde Escolar"); Ú5a - (Últimos 5 anos); NA: (Não é Artigo); NI - (Não tem no Idioma); NP - (Não é Pesquisa); NQ - (Não responde a Questão); NT - (Não é da Temática); ER - (Estudos Repetidos).

Fonte: Germano SNF, et al., 2020. 
Como critério de inclusão aplicou-se: artigos publicados na íntegra, disponíveis eletronicamente, em português, inglês e/ou espanhol, possuir aderência ao objetivo proposto. Foram excluídos estudos repetidos, revisões de literatura, estudos reflexivos e relatos. A análise das referências foi baseada nas publicações dos últimos cinco anos (de 2014 a 2019).

\section{RESULTADOS}

A busca nas bases de dados identificou 753.567 artigos acessados mencionando um dos descritores: Adolescente, Saúde Sexual e Reprodutiva, Tecnologia Educacional e Serviços de Saúde Escolar, após o cruzamento destes identificou-se 147 artigos que passaram por uma triagem aplicando-se os critérios de exclusão através da releitura dos títulos e resumos selecionados, excluindo-se 135 artigos, resultando 12 artigos que atenderam os critérios de inclusão da pesquisa e responderam à questão norteadora, estes foram lidos na integra fazendo parte da amostragem final da pesquisa (Figura 1).

Figura 1 - Fluxograma de seleção dos artigos para revisão integrativa. Manaus, AM, Brasil 2020.

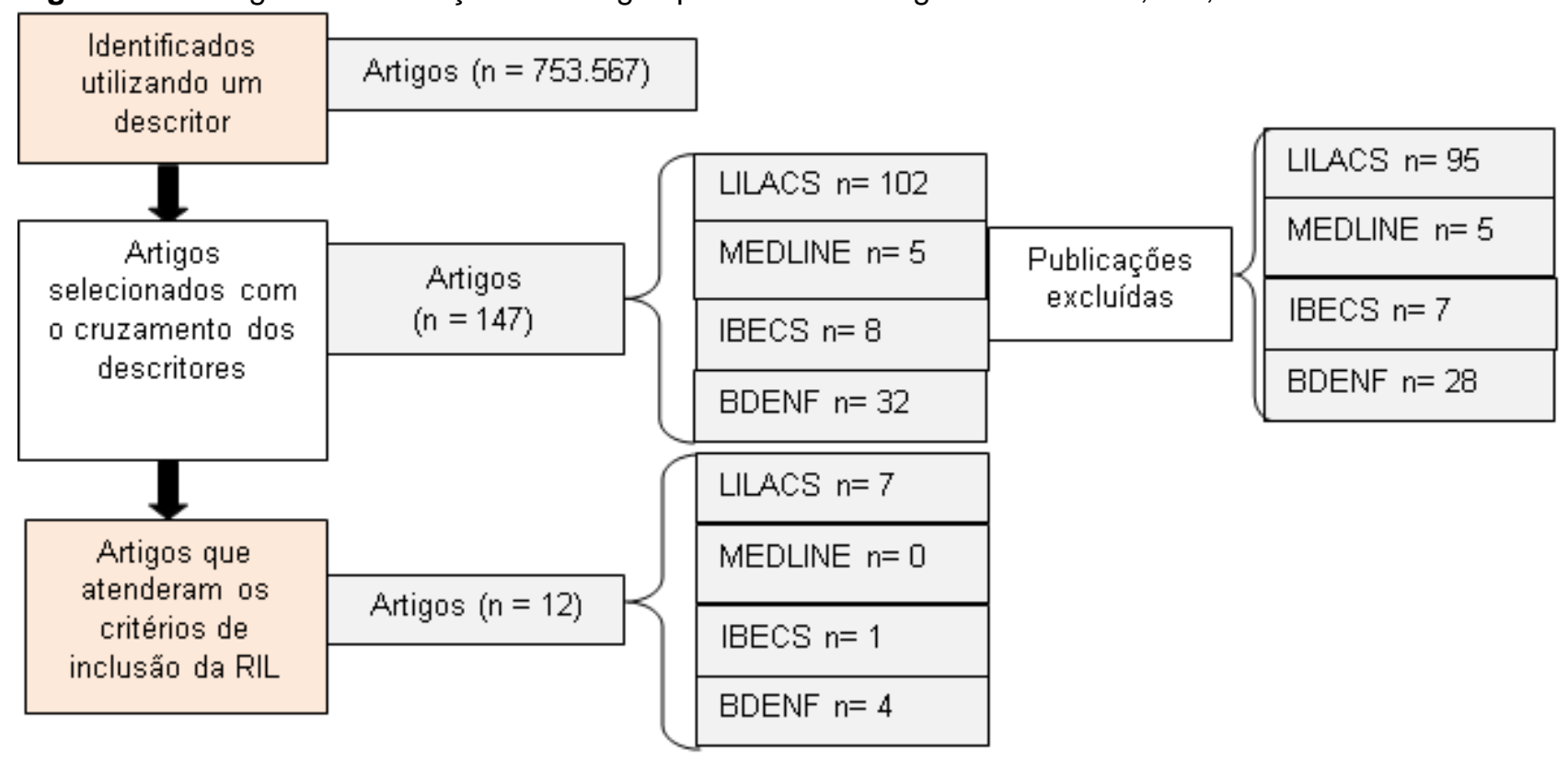

Fonte: Germano SNF, et al., 2020.

Com relação aos países de publicação, o Brasil surge com o maior número de artigos publicados nove (75\%), seguido do México com dois (16,6 \%) e a Colômbia com um (8,3\%). Demonstrando a escassez e a necessidade de mais publicações sobre este assunto em todos os países do mundo.

Quanto ao ano de publicação este estudo usou os últimos cinco anos de 2014 a 2019 como um dos critérios de inclusão, e 2016 foi o ano que mais foram publicados artigos que respondem a questão norteadora desta RIL totalizando quatro (33,3\%), depois vem 2015 com três artigos (25\%), 2017 e 2018 cada um com dois artigos somando quatro (33,3\%), 2019 um (8,3\%). Em 2014 não se identificou artigos publicados com relação à pergunta norteadora desta pesquisa.

Estes estudos foram publicados em periódicos nacionais e internacionais, a maioria deles nove foram publicados na área de Enfermagem (75\%), dois em Saúde pública/coletiva (16,6\%), e um na área de crescimento e desenvolvimento humano (8,3\%), destacando os periódicos da área da enfermagem como os principais em publicações nessa temática.

O panorama amostral constituída pelos artigos publicados com texto completo disponível de acordo com: base de dados, revista, título do artigo, autor(es), objetivo, natureza do estudo, país e ano de publicação que fazem parte desta pesquisa encontra-se no (Quadro 1), com sete estudos $58,3 \%$ da base de dados LILACS, um representando $8,3 \%$ da base de dados IBECS e quatro $33,3 \%$ da base de dados BDENF, destes estudos nove artigos (75\%) estão em português e três (25\%) em espanhol. 
Quadro 1 - Panorama dos artigos pesquisados de acordo com: base de dados, revista, título do artigo, autor (es), objetivo, natureza do estudo, país e ano. Manaus, AM, Brasil 2020.

\begin{tabular}{|c|c|c|c|c|c|c|c|c|}
\hline № & $\begin{array}{l}\text { Base de } \\
\text { dados }\end{array}$ & Revista & Titulo do artigo & Autor (es) & Objetivo & $\begin{array}{l}\text { Natureza do } \\
\text { Estudo }\end{array}$ & País & Ano \\
\hline 1 & LILACS & $\begin{array}{c}\text { Revista de } \\
\text { Enfermaría del } \\
\text { Instituto Mexicano }\end{array}$ & $\begin{array}{c}\text { Aconselhamento sobre Saúde } \\
\text { Sexual e Reprodutiva, estratégia } \\
\text { educacional de enfermagem para } \\
\text { reduzir riscos em adolescentes } \\
\text { grávidas. }\end{array}$ & $\begin{array}{l}\text { Martínez HT, } \\
\text { et al. (2019) }\end{array}$ & $\begin{array}{l}\text { Avaliar o efeito de uma } \\
\text { intervenção baseada em } \\
\text { "Aconselhamento Sexual em } \\
\text { Saúde e Reprodutiva. }\end{array}$ & $\begin{array}{c}\text { Desenho } \\
\text { experimental }\end{array}$ & México & 2019 \\
\hline 2 & LILACS & $\begin{array}{l}\text { Revista Ciência e } \\
\text { Cuidado }\end{array}$ & $\begin{array}{l}\text { Conhecimentos e práticas sobre } \\
\text { métodos de planejamento } \\
\text { família em adolescentes em idade } \\
\text { escolar de Cartagena-Bolívar. }\end{array}$ & $\begin{array}{l}\text { Del Toro RM, } \\
\text { et al. (2018) }\end{array}$ & $\begin{array}{l}\text { Identificar o conhecimento e a } \\
\text { prática dos métodos de } \\
\text { planejamento familiar aos } \\
\text { alunos de uma instituição } \\
\text { educacional. }\end{array}$ & $\begin{array}{l}\text { Descritivo, } \\
\text { transversal e } \\
\text { correlacional }\end{array}$ & Colômbia & 2018 \\
\hline 3 & LILACS & Saúde e socieda & $\begin{array}{l}\text { Eu virei homem! : a construção das } \\
\text { masculinidades para adolescentes } \\
\text { participantes de um projeto de } \\
\text { promoção de saúde sexual e } \\
\text { reprodutiva }\end{array}$ & $\begin{array}{l}\text { Vasconcelos } \\
\text { ACS, et al. } \\
(2016)\end{array}$ & $\begin{array}{l}\text { Compreender e discutir as } \\
\text { construções sobre as } \\
\text { masculinidades no discurso de } \\
\text { adolescentes. }\end{array}$ & $\begin{array}{l}\text { Qualitativa, } \\
\text { Exploratório }\end{array}$ & Brasil & 2016 \\
\hline 4 & LILACS & $\begin{array}{l}\text { Revista eletrônica } \\
\text { de enfermagem }\end{array}$ & $\begin{array}{l}\text { Conhecimento em saúde sexual e } \\
\text { reprodutiva: estudo transversal com } \\
\text { adolescentes }\end{array}$ & $\begin{array}{l}\text { Oliveira PC, } \\
\text { et al. (2017) }\end{array}$ & $\begin{array}{c}\text { Investigar e comparar o } \\
\text { conhecimento em saúde sexual } \\
\text { e reprodutiva. }\end{array}$ & $\begin{array}{l}\text { Estudo de } \\
\text { corte } \\
\text { transversal }\end{array}$ & Brasil & 2017 \\
\hline 5 & LILACS & $\begin{array}{c}\text { Interface - } \\
\text { Comunicação } \\
\text { Saúde, Educação }\end{array}$ & $\begin{array}{l}\text { A intersetorialidade como } \\
\text { estratégia para promoção da saúde } \\
\text { sexual e reprodutiva dos } \\
\text { adolescentes. }\end{array}$ & $\begin{array}{l}\text { Higa EFR, et } \\
\text { al. (2015). }\end{array}$ & $\begin{array}{l}\text { Analisar se as escolas estão } \\
\text { desenvolvendo ações para } \\
\text { promoção da saúde sexual e } \\
\text { reprodutiva dos adolescentes. }\end{array}$ & $\begin{array}{l}\text { Qualitativo } \\
\text { do tipo } \\
\text { descritivo. }\end{array}$ & Brasil & 2015 \\
\hline 6 & LILACS & $\begin{array}{l}\text { Journal of Human } \\
\text { Growth and } \\
\text { Development }\end{array}$ & $\begin{array}{l}\text { Acessibilidade dos adolescentes às } \\
\text { fontes de informações sobre saúde } \\
\text { sexual e reprodutiva. }\end{array}$ & $\begin{array}{l}\text { Gondim PS, } \\
\text { et al. (2015) }\end{array}$ & $\begin{array}{c}\text { Identificar as fontes de } \\
\text { informações utilizadas pelos } \\
\text { adolescentes da escola pública } \\
\text { sobre sexualidade e reprodução }\end{array}$ & $\begin{array}{l}\text { Qualitativo } \\
\text { do tipo } \\
\text { descritivo. }\end{array}$ & Brasil & 2015 \\
\hline
\end{tabular}




\section{Revista Eletrônica Acervo Saúde / Electronic Journal Collection Health | ISSN 2178-2091}

\begin{tabular}{|c|c|c|c|c|c|c|c|c|}
\hline № & $\begin{array}{l}\text { Base de } \\
\text { dados }\end{array}$ & Revista & Titulo do artigo & Autor (es) & Objetivo & $\begin{array}{l}\text { Natureza do } \\
\text { Estudo }\end{array}$ & País & Ano \\
\hline 7 & LILACS & $\begin{array}{l}\text { Revista Minera de } \\
\text { Enfermagem }\end{array}$ & $\begin{array}{l}\text { Comportamento sexual de } \\
\text { adolescentes escolares }\end{array}$ & $\begin{array}{l}\text { Silva GS, et } \\
\text { al. (2015) }\end{array}$ & $\begin{array}{l}\text { Descrever as situações } \\
\text { relacionadas à saúde sexual } \\
\text { dos adolescentes. }\end{array}$ & $\begin{array}{l}\text { Descritivo, } \\
\text { transversal }\end{array}$ & Brasil & 2015 \\
\hline 8 & IBECS & $\begin{array}{l}\text { REUOL Revista } \\
\text { de Enfermagem } \\
\text { UFPE Online }\end{array}$ & $\begin{array}{c}\text { Tecnologia Educacional Baseada } \\
\text { em Nola Pender: Promoção da } \\
\text { Saúde do Adolescente }\end{array}$ & $\begin{array}{l}\text { Santos AS, } \\
\text { et al. (2018) }\end{array}$ & $\begin{array}{c}\text { Apresentar uma tecnologia } \\
\text { educativa em Enfermagem, } \\
\text { voltada para adolescentes, } \\
\text { acerca da vacinação contra o } \\
\text { HPV. }\end{array}$ & $\begin{array}{l}\text { Qualitativo } \\
\text { do tipo } \\
\text { descritivo. }\end{array}$ & Brasil & 2018 \\
\hline 9 & BDENF & $\begin{array}{l}\text { Revista de } \\
\text { Enfermaría del } \\
\text { Instituto } \\
\text { Mexicano. }\end{array}$ & $\begin{array}{l}\text { Intervenção educacional de } \\
\text { enfermagem em saúde sexual e } \\
\text { reprodutivaem adolescentes }\end{array}$ & $\begin{array}{l}\text { Becerril JR, } \\
\text { et al. (2016) }\end{array}$ & $\begin{array}{l}\text { Projetar um programa de } \\
\text { enfermagem em educação } \\
\text { sexual e reprodutiva com } \\
\text { adolescentes e suas famílias. }\end{array}$ & $\begin{array}{l}\text { Estudo de } \\
\text { intervenção }\end{array}$ & México & 2016 \\
\hline 10 & BDENF & $\begin{array}{c}\text { Revista de } \\
\text { Enfermagem da } \\
\text { UFPI }\end{array}$ & $\begin{array}{l}\text { Potencialidades e desafios na } \\
\text { realização de oficinas educativas } \\
\text { com adolescentes }\end{array}$ & $\begin{array}{l}\text { Morais RS, } \\
\text { et al. (2017) }\end{array}$ & $\begin{array}{l}\text { Identificar os fatores } \\
\text { potencializadores e desafios na } \\
\text { realização das oficinas } \\
\text { educativas. }\end{array}$ & $\begin{array}{l}\text { Qualitativa } \\
\text { do tipo } \\
\text { descritivo. }\end{array}$ & Brasil & 2017 \\
\hline 11 & BDENF & $\begin{array}{l}\text { REUOL Revista } \\
\text { de Enfermagem } \\
\text { UFPE Online }\end{array}$ & $\begin{array}{c}\text { Validação de um material educativo } \\
\text { como ferramenta pedagógica para } \\
\text { adolescentes sobre iniciação } \\
\text { sexual }\end{array}$ & $\begin{array}{l}\text { Ferreira AS, } \\
\text { et al. (2016) }\end{array}$ & $\begin{array}{l}\text { Validar a história em quadrinhos } \\
\text { “Iniciação sexual: Já estou } \\
\text { pronto para iniciar minha vida } \\
\text { sexual?”. }\end{array}$ & $\begin{array}{c}\text { Pesquisa } \\
\text { metodológica }\end{array}$ & Brasil & 2016 \\
\hline 12 & BDENF & $\begin{array}{l}\text { REUOL Revista } \\
\text { de Enfermagem } \\
\text { UFPE Online }\end{array}$ & $\begin{array}{c}\text { Validação de material educativo } \\
\text { como ferramenta pedagógica sobre } \\
\text { métodos contraceptivos para } \\
\text { adolescentes }\end{array}$ & $\begin{array}{l}\text { Almeida TG, } \\
\text { et al. (2016) }\end{array}$ & $\begin{array}{c}\text { Validar o material educativo } \\
\text { "Métodos Contraceptivos: Sou } \\
\text { jovem e ainda não quero gerar } \\
\text { uma vida!". }\end{array}$ & $\begin{array}{c}\text { Pesquisa } \\
\text { metodológica }\end{array}$ & Brasil & 2016 \\
\hline
\end{tabular}

Fonte: Germano SNF, et al., 2020. 
A maioria dos estudos possui a natureza qualitativo cinco $(41,6 \%)$, descritivo transversal dois $(16,6 \%)$, mesmo percentual da pesquisa metodológica dois $(16,6 \%)$, desenho experimental um $(8,3 \%)$, estudo de corte um $(8,3 \%)$ e estudo de intervenção um $(8,3 \%)$.

Dos 12 artigos utilizados na RIL, sete (58,3\%) trouseram intervenções tecnologicas educativas para saúde sexual e reprodutiva dos adolescente que são/ou podem ser aplicadas nas escolas públicas, três (25\%) abordaram o conhecimento dos adolescentes de escola pública sobre saúde sexual e reprodutiva, enfatizando a necessidade desta temática ser trabalhada em todas as escolas, e dois (16,6\%) trouseram as potencialidades e fragilidades para a educação sexual e reprodutivas de adolescentes.

\section{DISCUSSÃO}

Para apresentação da discussão dos resultados desta pesquisa, os estudos foram agrupados em três categorias conforme as estratégias identificadas: Tecnologias educativas para saúde sexual e reprodutiva de adolescentes nas escolas públicas; o conhecimento dos adolescentes de escola pública sobre saúde sexual e reprodutiva; e as potencialidades e fragilidades para a educação sexual e reprodutivas de adolescentes nas escolas públicas, discutidos a seguir.

\section{Tecnologias educativas para saúde sexual e reprodutiva de adolescentes nas escolas publicas}

Os artigos selecionados nas bases de dados trazem variadas TEs para trabalhar a saúde sexual e reprodutiva dos adolescentes de escolas públicas. Um estudo analisou a utilização de jogos educativos (quebra cabeças) como TEs com 24 adolescentes, demonstrando que as tecnologias em formato de jogos educativos são muito aceitas pelos adolescentes por tornarem eles sujeitos ativos no processo de aprendizado, tornando estes mediadores na educação em saúde contemplando os vários temas relacionado ao corpo masculino e feminino, infecções sexualmente transmissíveis IST`s e gravidez na adolescência, além de outros assuntos que são sugeridos por eles (VASCONCELOS ACS, et al., 2016).

Outra TE relatada nos artigos foi o (Teatro e Dinâmica Verdade ou Mito) como instrumento de educação em saúde abordando questões que envolvem papilomavírus humano (HPV), em escolas municipais vinculadas à Estratégia de Saúde da Família do município de Juazeiro do Norte, a partir do Programa de Saúde na Escola, espaço onde os adolescentes esclareceram o conhecimento prévio que tinham e tiraram dúvidas num contexto de aprendizado dinâmico (SANTOS AS, et al., 2018).

A peça teatral e a dinâmica foram escritas pela pesquisadora utilizando uma teoria de enfermagem adequada ao contexto do Nordeste brasileiro. Isso atraiu a atenção dos adolescentes, por se tratar de uma atividade educativa que permitiu a participação ativa dos sujeitos envolvidos, possibilitando o esclarecimento de dúvidas, além de paralelamente, oportunizar mapear equívocos no conhecimento dos adolescentes (SANTOS AS, et al., 2018).

Através do estudo sobre a preparação de um material educacional para aconselhamento sobre saúde sexual e reprodutiva com adolescentes grávidas, demostrou-se que a frequência de complicações no grupo de intervenção com as adolescentes grávidas foi positiva versus o grupo controle, reduzindo os riscos das adolescentes durante o parto e pós-parto que fizeram parte do grupo de intervenção sobre: cuidados no final da gravidez, direitos sexuais e reprodutivos, fatores de risco associados à depressão em adolescentes grávidas, violência na adolescente grávida e amamentação (MARTíNEZ HT, et al., 2019).

Em uma escola pública foi realizada entrevistas com 90 adolescentes, na faixa etária entre 16 e 19 anos, estes demonstraram o interesse por diversas tecnologias como televisão (filme, series e vídeos) (35,5\%), seguido da internet, livros e revistas como fonte de informação sobre saúde sexual e reprodutiva, destes 62 adolescentes (68,9\%) afirmaram já terem participado de atividade educativa utilizando alguma destas tecnologias na escola (GONDIM PS, et al., 2015).

Porém é importante que os conteúdos das variadas TEs, jogos, teatro, televisão, internet, livros, e revistas bem como demais tecnologias, precisam ser adequados a faixa etária e o contexto sociocultural da população, demonstrando a importância da validação das TEs por juízes especialistas na temática e na faixa etária, antes destas serem utilizadas no contexto escolar para educação desta população (ALMEIDA TG, et al., 2016). 
Atendendo essa necessidade algumas das pesquisas trouxeram a validação de TEs para os adolescentes, uma delas trouxe a validação da aparência e conteúdo de uma história em quadrinhos intitulada "Iniciação sexual: já estou pronto para iniciar minha vida sexual?", como tecnologia para a promoção da saúde sexual e reprodutiva de adolescentes, através de uma pesquisa metodológica com juízes especialista na temática que validaram o material, para ser utilizado na construção do conhecimento dos adolescentes de forma segura e dialogada com os professores (FERREIRA AS, et al., 2016).

Outra pesquisa, também validou com juízes especialistas na temática sexualidade e reprodução na adolescência, a TE "Métodos Contraceptivos: sou jovem e ainda não quero gerar uma vida!", no formato de história em quadrinhos, como ferramenta pedagógica otimizando o processo de ensino aprendizagem da sexualidade de meninos e meninas adolescentes, permitindo também que estes tornem-se multiplicadores de conhecimento através da TE com aqueles que não tiveram acesso a esta ferramenta (ALMEIDA TG, et al., 2016).

Porém outro estudo, desenvolvido em vinte escolas públicas no interior paulista, para analisar as principais ações desenvolvidas por estas, utilizando TEs para promoção da saúde sexual e reprodutiva dos adolescentes, evidenciou-se que o uso destas, foi citado, somente por $30 \%$ das escolas, que usavam como TEs peças anatômicas e urnas de dúvidas, foram citadas ainda, que somente $25 \%$ das escolas, usavam recursos interativos e audiovisuais, os quais despertam grande interesse entre os jovens (HIGA EFR, et al., 2015).

O contexto analisado mostra uma variada gama de TEs, que muitas escolas públicas ainda não se apropriaram para trabalhar a saúde sexual e reprodutiva dos adolescentes em parceira com os serviços de saúde, realizando desta maneira a educação dos adolescentes de forma limitada e pouco ativa, colocando eles como meros espectadores de conteúdos excessivamente monótonos, tornando estes indivíduos pouco críticos, não atendendo as propostas educacionais das políticas públicas de promoção da saúde sexual e reprodutiva dos adolescentes nas escolas.

\section{O conhecimento que os adolescentes de escola pública apresentam sobre saúde sexual e reprodutiva}

Ao realizar a análise das TEs utilizadas na educação sexual e reprodutiva dos adolescentes de escolas públicas, alguns artigos trazem os conhecimentos que estes apresentam sobre a temática antes e após a utilização destas tecnologias, evidenciando que alguns conhecimentos são adquiridos de forma aleatória e fragmentado através de seus parceiros e colegas, tornando estes vulneráveis a conhecimentos incorretos, 0 que leva a práticas inseguras na vida sexual e reprodutiva destes adolescentes.

Um estudo de intervenção realizado com 38 adolescentes de 12 aos 19 anos para conhecer as informações que estes possuíam para implementar um programa de enfermagem em educação sexual e reprodutiva no Estado do México, demonstrou-se que os adolescentes possuíam conhecimentos limitados e confusos, acreditavam que tanto o preservativo quanto pílulas fornecem proteção para prevenir as ISTs e a gravidez.

Constatou-se também neste estudo que antes da intervenção educativa 52,6\% dos adolescentes identificaram apenas AIDS / HIV como IST mais conhecida; não mencionando as demais, herpes, gonorreia, sífilis e papilomavírus humano (HPV), demonstrando que a realização de programas educacionais são necessários para o conhecimento e promoção da saúde desta população (BECERRIL JR, et al., 2016).

Outro estudo analítico de corte transversal, realizado com 2.449 adolescentes de 12 a 18 anos de sete escolas públicas vinculadas ao serviço de saúde em Goiânia, trouxe o mesmo dado preocupante, que embora um número significativo de adolescentes apresentassem algum nível de conhecimento sobre as ISTs, apenas a AIDS e hepatite $B$ são relatadas por mais da metade destes, evidenciando o desconhecimento quanto às outras infecções, que apresentam elevadas e relevantes complicações sexuais e reprodutivas (OLIVEIRA PC, et al., 2017).

Porém o estudo composto por 229 adolescentes de uma escola pública, que receberam informações sobre métodos contraceptivos na escola pelos professores e profissionais da saúde, ao participarem da pesquisa utilizando um instrumento intitulado "conhecimento e práticas sobre métodos de planejamento familiar", $86 \%$ 
destes afirmaram que o único método contraceptivo que impede tanto gravidez como as doenças sexualmente transmissíveis é o preservativo; e reconhecem que na primeira relação sexual pode-se engravidar $(71,2 \%)$ (DEL TORO RM, et al., 2018).

Os estudos trazem também diferenças estatísticas entre os sexos, em relação à saúde sexual e reprodutiva, evidenciando o sexo feminino com maior conhecimento sobre métodos contraceptivos hormonais e naturais, já o preservativo masculino não apresentou significância estatística, visto que ambos os sexos já o conheciam, o fato de o sexo feminino ser detentor de mais conhecimento nessa área, pode ser pelo fato de as mulheres culturalmente serem responsáveis pela saúde da família e as mesmas procurarem mais os serviços de saúde que os homens (OLIVEIRA PC, et al., 2017).

As temáticas em saúde sexual e reprodutiva deveriam ser abordadas de forma cotidiana por profissionais das áreas da saúde e educação junto aos adolescentes nas escolas. Apesar de o tema ser considerado simples e cotidiano, o conhecimento de adolescentes acerca da temática ainda apresenta-se incipiente, como demonstrado nos estudos, o que pode contribuir para comportamentos inadequados inseguros e práticas sexuais de risco (DEL TORO RM, et al., 2018). Nota-se assim a necessidade de sensibilizar alguns profissionais da saúde, educadores e familiares a trabalharem em parceria no âmbito escolar a temática sexualidade e reprodução, pois, apesar desta temática ser relevante para o desenvolvimento integral da saudável dos adolescentes está ainda é incipiente.

\section{As potencialidades e fragilidade para a educação sexual e reprodutivas dos adolescentes nas escolas públicas}

A maioria dos artigos desta pesquisa de revisão de literatura citou em algum momento as potencialidades e dificuldades de se trabalhar essa temática, porém dois artigos (16,6\%) trouseram como temática principal as potencialidades e fragilidades de se trabalhar a educação sexual e reprodutiva dos adolescentes em escolas públicas.

Um destes artigos descreveu uma pesquisa desenvolvida junto ao Projeto Flor do Mandacaru, no município de Sobral, Ceará, através da análise das atividades desenvolvidas com os adolescentes sobre saúde sexual e reprodutiva, identificando como potencialidade o espaço escolar como local mais apropriado e onde os adolescentes estão abertos para discutir a temática, bem como a visão dos profissionais quanto a importância de se trabalhar a temática na escola com a equipe multidisciplinar (MORAIS RS, et al., 2017).

No estudo transversal desenvolvido em todas as escolas estaduais do município de Diamantina- MG, Brasil, com 323 adolescentes do $9^{\circ}$ ano do ensino fundamental e $1^{\circ}, 2^{\circ}$ e $3^{\circ}$ anos do ensino médio, trouxe como potencialidade o reconhecimento dos profissionais das instituições quanto à educação em saúde método principal para trabalhar a sexualidade dos adolescentes de escolas públicas, bem como 0 reconhecimento em todo o mundo da importância de se trabalhar essa temática com adolescentes escolares, tendo apoio dos governos através dos setores Saúde e Educação implantando e trabalham projetos de Saúde e Prevenção nas escolas, desenvolvendo ações voltadas para a redução da gravidez na adolescência e dos índices de ISTs nesta população (SILVA GS, et al., 2015).

Porém, assim como temos potencialidades temos fragilidades, e uma delas é há resistência para a realização da educação em saúde sobre sexualidade e reprodução com os adolescentes escolares em virtude do estigma de se trabalhar essa temática com essa população em algumas escolas, bem como a escassez de recursos tecnológicos educacionais atrativos disponíveis nas escolas públicas para se trabalhar com esses adolescentes em oficinas mais didáticas e dinâmicas (MORAIS RS, et al., 2017).

O comportamento sexual dos adolescentes é caracterizado por várias atitudes que põem em risco a sua saúde, e isso se deve a vários fatores que fragiliza o trabalho com os adolescentes de escola pública como: exposição a álcool e drogas; ausência de parceria pais e escola para trabalhar esta temática, despreparo destes para educar os adolescentes sobre o assunto; fragilidade no vínculo e acolhimento efetivo dos adolescentes nos serviços de saúde; despreparo das escolas e dos profissionais da educação e até mesmo da saúde; o acesso a informações inadequadas e até distorcidas pela mídia (SILVA GS, et al., 2015). 
Além disso, apesar da inserção dos profissionais de saúde da atenção primária para realização de atividades educativas sobre saúde sexual e reprodutiva nas escolas públicas em parceria com os professores, estas atividades ainda são pouco frequentes e descontinuas, um dos motivos é os interesses conflitantes entre os professores, pais e alunos sobre esta temática. Muitas vezes os pais querem que os professores tenham a didática voltada mais para a educação moral e tradicional dos adolescentes, fragilizando a promoção da saúde sexual e reprodutiva nas escolas com os adolescentes (MORAIS RS, et al., 2017).

A família é o eixo central que influência a saúde sexual e reprodutiva podendo potencializar as ações de educação em saúde nesta temática ou fragilizar, já que está representa a estrutura social relevante para a educação de seus filhos, especialmente no tocante da sexualidade. Entretanto, ainda se encontram dados que mostram a sensação de despreparo dos pais para atuar na educação sexual e reprodutiva dos filhos, isso acontece devido às dificuldades encontradas em abordar o assunto, decorrente da falta de informações ou por vergonha de abordar a temática (SILVA GS, et al., 2015).

As fragilidades destacadas fazem com que os adolescentes se afastem dos profissionais da educação e da saúde para esclarecer suas dúvidas sobre sexo, sexualidade e reprodução recorrendo aos seus familiares, parceiros e amigos que muitas vezes não estão preparados para o esclarecimento correto destas dúvidas, deixando os adolescentes sem acesso as informações corretas e vivências mais apropriadas (MORAIS RS, et al., 2017).

Como limitações deste estudo destacam-se a falta de abordagem sobre as TEs sobre saúde sexual e reprodutiva nas escolas particulares, bem como a comparação entre estas e as escolas públicas, ficando estas limitações como ideias a serem exploradas em outras pesquisas a serem realizadas.

\section{CONSIDERAÇÕES FINAIS}

Na literatura foi identificada uma variada quantidade de TE sobre saúde sexual e reprodutiva, que devem ser utilizadas na promoção da saúde, prevenção das ISTS e gravidez indesejada na adolescensia, enfatizando que o ambiente escolar é mais propício a se produzir e trabalhar estas tecnologias. Porém, ainda há muitas instituições públicas de ensino que não trabalham com TE nesta temática por apresentarem algumas fragilidades como, falta de material adequado disponibilizado pela escola e ausência de educação permanente dos professores e profissionais da saúde para saber lidar com esta temática que ainda é tratada com estigma e preconceitos por eles próprios, acarretando em falhas na educação sexual e reprodutiva dos adolescentes de escolas públicas tornando estes vulneráveis a gravidez indesejada e as ISTs.

\section{REFERÊNCIAS}

1. ALMEIDA TG, et al. Validação de material educativo como ferramenta pedagógica sobre métodos contraceptivos para adolescentes. Rev. Enferm. UFPE, 2016; 10(12): 4696-700.

2. BECERRIL JR, et al. Intervención educativa de enfermería sobre salud sexual y reproductiva en adolescentes. Rev. Enferm. Inst. Mex Seguro Soc, 2016; 24(1):514.

3. BRASIL. Ministério da Saúde. Secretaria de Atenção à Saúde. Proteger e cuidar da saúde de adolescentes na atenção básica. Departamento de Ações Programáticas e Estratégicas. Biblioteca virtual da saúde, 2018. 233p.

4. DEL TORO RM, et al. Conocimientos y prácticas sobre métodos de planificación familiar en adolescentes escolarizados de Cartagena-Bolívar. Rev. cienc. Cuidad, 2018; 15(2): 24-37.

5. DONATO H, DONATO M. Etapas na Condução de uma Revisão Sistemática. Revista Científica da Ordem dos Médicos, 2019; 32(3):227-235.

6. FERREIRA AS, et al. Validação de um material educativo como ferramenta pedagógica para adolescentes sobre iniciação sexual. Rev. Enferm. UFPE, 2016; 10(Supl. 5):4412-5.

7. FIEDLER MW, et al. A prevenção da gravidez na adolescência na visão deadolescentes. Texto Contexto Enferm, 2015; 24(1): 30-7.

8. GONÇALVES LFF, et al. Promoção de saúde com adolescentes em ambiente escolar: relato de experiência. SANARE, 2016.

9. GONDIM PS, et al. Acessibilidade dos adolescentes às fontes de informações sobre saúde sexual e reprodutiva. Journal of Human Growt hand Development, 2015; 25(1): 50-53.

10. HIGA EFR, et al. A intersetorialidade como estratégia para promoção da saúde sexual e reprodutiva dos adolescentes. Interface-Comunicação, Saúde, Educação, 2015; 19 Supl 1:879-91.

11. MARTÍNEZ HT, et al. Consejería em Salud Sexual y Reproductiva, estrategia educativa de enfermería para reducir adolescentes embarazadas. Rev Enferm. Inst. Mex. Seguro Soc, 2019; 27(1): 23-32. 
12. MORAIS RS, et al. Potencialidades e desafios na realização de oficinas educativas com adolescentes. Rev. Enferm. UFPI, 2017; 6(2): 30-6 ISSN: 2238-7234.

13. NETO JMR, et al. Meleis' Nursing Theories Evaluation: integrative review. Rev Bras Enferm, 2016; 69(1):162-8.

14. OLIVEIRA PC, et al. Conhecimento em saúde sexual e reprodutiva: estudo transversal com adolescentes. Rev. Eletr. Enf, 2017;19:a17.

15. SANTOS AS, et al. Tecnologia educacional baseada em Nola Pender: promoção da saúde do adolescente. Revenferm UFPE, 2018;12(2):582-8.

16. SFAIR SC, et al. Educação sexual para adolescentes e jovens: mapeando proposições oficiais. Saúde Soc, 2015 ; p.620-632.

17. SILVA DML, et al. Tecnologias educacionais na assistência de enfermagem em educação em saúde: revisão integrativa. Rev. Enferm. UFPE, 2017; 2):1044-51.

18. SILVA GS, et al. Comportamento sexual de adolescentes escolares. REME Rev. Min. Enferm, 2015; 19(1): 154-160.

19. VASCONCELOS ACS, et al. Eu virei homem!: a construção das masculinidades para adolescentes participantes de um projeto de promoção de saúde sexual e reprodutiva. Saúde Soc, 2016; n.1, p.186-197. 